\title{
Temperature difference measurement with using two RTD sensors as example of evaluating uncertainty of a vector output quantity
}

\author{
Adam Idzkowski', Zygmunt Warsza ${ }^{2}$ \\ ${ }^{1}$ Faculty of Electrical Engineering, Bialystok University of Technology, Bialystok, Poland \\ ${ }^{2}$ Lukasiewicz Research Network - Industrial Research Institute of Automation and Measurements PIAP, \\ Warszawa, Poland \\ ${ }^{1}$ Corresponding author \\ E-mail: ${ }^{1}$ a.idzkowski@pb.edu.pl, ${ }^{2}$ zlw1936@gmail.com
}

Received 30 June 2021; received in revised form 15 September 2021; accepted 27 October 2021 DOI https://doi.org/10.21595/rsa.2021.22143

Check for updates

Copyright (C) 2021 Adam Idzkowski, et al. This is an open access article distributed under the Creative Commons Attribution License, which permits unrestricted use, distribution, and reproduction in any medium, provided the original work is properly cited.

\begin{abstract}
The evaluation of results in the case of indirect multi-parameter measurements is presented. A theoretical basis for determining the estimates of values, the uncertainties and the correlation coefficients of indirectly obtained multi-measurand is explained in detail. The example of a difference and an average of two-temperature indirect measurement is given with the use of two RTDs. Such sensors are used in the laboratory and industrial temperature control systems. The uncertainty of measurements depends on the errors of two RTDs and the structure of signal conditioning circuit.
\end{abstract}

Keywords: temperature sensors, RTDs, measurement uncertainty, multivariate measurements, mathematical modeling.

\section{Introduction}

Temperature is the most frequently measured parameter in healthcare, industrial, air conditioning and ventilation, robotic and automotive applications [1], [2]. To know temperature or temperature difference with the right balance of accuracy, precision and repeatability is critical in many applications [3]. Commonly used in temperature measurement are resistance temperature detectors (RTDs) - precise metal components usually made of pure or almost pure platinum. The platinum sensor (e.g. Pt100) has a fully defined, repeatable and characterized resistance vs. temperature function. Therefore, RTD sensors are widely used in scientific and instrumentation applications. Moreover, many applications require multiple RTDs, so the interface and associated circuitry must also be appropriate for the application [4], [5].

Standard platinum RTD sensors (IEC 60751) operate in the range of $-200{ }^{\circ} \mathrm{C}$ to $+800{ }^{\circ} \mathrm{C}$. They are properly driven by a current or voltage source [6]. Their resistance is measured as a voltage across two terminals with an appropriate analog front-end (AFE) circuit, with the voltage reading it is linearized for the highest accuracy. Lead-wire-resistance compensation techniques are used.

The simultaneous temperature difference $t_{D}$ and average $t_{a v}$ readings can be taken as an example of the multivariate measurement [7]. A Wheatstone bridge-circuit, classically supplied, can be used for measuring temperature, i.e. with one or two resistance temperature detectors (RTDs). It is widely presented in publication [8] where the values of limited errors and expanded uncertainties are calculated for a few variants of this circuit with the Class A and B industrial Pt100 sensors. However, this approach assumed that input and output measurands were uncorrelated. In this article the correlated measurands are considered.

Having transfer function of a bridge-circuit, the descriptive statistics which bases on evaluating uncertainty, can be performed. It is based on a vector method for determining uncertainty of multivariate indirect measurements, which is presented in Supplement 2 to the GUM Guide [9]. In this paper such approach is presented. 
Propagation of measurement uncertainty using covariance matrix assumes that the input measurand is formulated by a random vector $\mathbf{X}=\left[X_{1}, X_{2}, \ldots, X_{m}\right]^{T}$ and the output measurand by a random vector $\mathbf{Y}=\left[Y_{1}, Y_{2}, \ldots, Y_{n}\right]^{T}$. The vectors of dimensions $m$ and $n$ represent the multi-dimensional distributions of $\mathbf{X}$ and $\mathbf{Y}$. If $f$ is a linear operator, then $n \leq m$. The relation between them is presented as a generalized equation [10]:

$\mathbf{Y}=f^{\prime}(\mathbf{h})=f(\mathbf{X})$

where $f$ describes the system equation as a function of the input quantities, while $f^{\prime}$ is a function of the uncorrelated subsystems. Vector $\mathbf{h}$ contains the uncertainties of every subsystem $\mathbf{h}=\left[\mathbf{h}_{1}^{T}, \ldots, \mathbf{h}_{K}^{T}\right]^{T}$. Symbol $K$ represents the number of subsystems [11].

The relation between covariance matrices $\mathbf{c}_{\mathbf{X}}, \mathbf{c}_{\mathbf{Y}}$ of the output measurand $\mathbf{Y}$ and input measurand $\mathbf{X}$ is expressed as:

$\mathbf{c}_{\mathbf{Y}}=\mathbf{S} \cdot \mathbf{c}_{\mathbf{X}} \cdot \mathbf{S}^{T}$

where $\mathbf{S} \equiv \partial \mathbf{Y} / \partial \mathbf{X}$ is the matrix of sensitivity coefficients [12]. The model Eq. (2) is true if it is either linear or can be linearized in the working point using a Taylor series. Usually, the uncertainties for the input parameters are small. Another assumption is that the probability density functions (PDFs) should be symmetric and common.

A covariance matrix $\boldsymbol{c}$ and a correlation matrix $\mathbf{K}$ are defined by:

$\mathbf{c}=\boldsymbol{\sigma} \cdot \mathbf{K} \cdot \boldsymbol{\sigma}^{T}$,

where $\boldsymbol{\sigma}$ is the diagonal matrix of standard deviations [10].

\section{Average and difference temperature measurement - circuits, transfer functions, uncertainty}

There are many designs of analog front-end circuits for RTDs measurement. In Fig. 1(a) classical Wheatstone bridge-circuit is presented. Another circuit (Fig. 1(b)) differs from the previous one, i.e. output voltages are proportional to a difference and a sum of temperatures $t_{1}$, $t_{2}$, starting with the initial temperature $t_{0}$. This circuit is the alternative to the classical bridge-circuit. Both circuits were described in previous articles of authors [8], [13]. Below, the uncertainty propagation will be expressed using model Eq. (2).
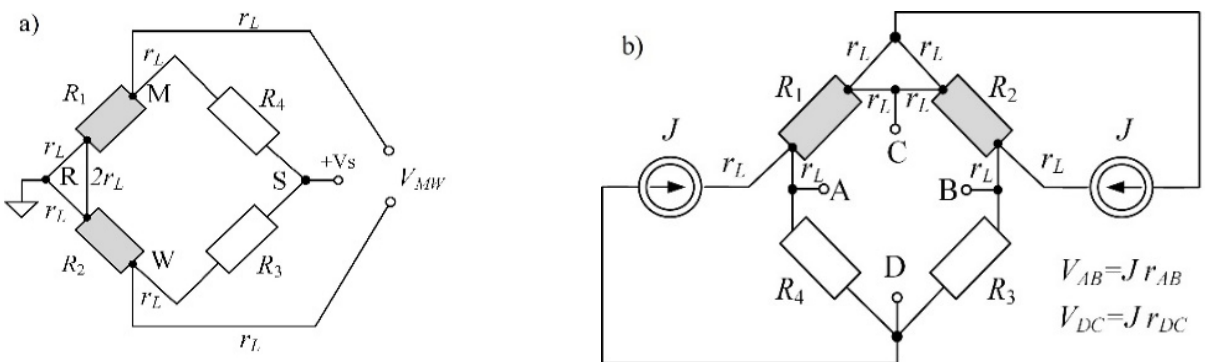

Fig. 1. a) The classical and unconventional, b) bridge-circuits as resistance to voltage converters, the resistances $R_{1}$ and $R_{2}$ represent two RTDs

Two transfer resistances $r_{A B}=f_{1}\left(t_{D}\right), r_{C D}=f_{2}\left(t_{a v}\right)$ of the bridge-circuit depend on the resistances $R_{1} \ldots R_{4}$ in different way (assuming that constant currents $J$ are equal):

$r_{A B}=\frac{V_{A B}}{J}=\frac{R_{1} R_{4}-R_{2} R_{3}}{\sum R_{i}}, \quad r_{C D}=\frac{V_{C D}}{J}=\frac{R_{1} R_{2}-R_{3} R_{4}}{\sum R_{i}}$, 
where: $\Sigma R_{i}=R_{1}+R_{2}+R_{3}+R_{4}$, lead resistances $r_{L} \ll R_{1}, R_{2}, R_{3}, R_{4}$.

An input matrix $\mathbf{c}_{\mathbf{X}}$ contains only variances (squared uncertainties) because it is assumed that the input variables are uncorrelated and normally distributed:

$\mathbf{c}_{\mathbf{X}}\left(R_{1}, R_{2}, R_{3}, R_{4}\right)=\left[\begin{array}{cccc}\sigma_{R 1}^{2} & 0 & 0 & 0 \\ 0 & \sigma_{R 2}^{2} & 0 & 0 \\ 0 & 0 & \sigma_{R 3}^{2} & 0 \\ 0 & 0 & 0 & \sigma_{R 4}^{2}\end{array}\right]$

The sensitivity matrix is as follows:

$\mathbf{S}=\left[\begin{array}{llll}\frac{\partial r_{A B}}{\partial R_{1}} & \frac{\partial r_{A B}}{\partial R_{2}} & \frac{\partial r_{A B}}{\partial R_{3}} & \frac{\partial r_{A B}}{\partial R_{4}} \\ \frac{\partial r_{C D}}{\partial R_{1}} & \frac{\partial r_{C D}}{\partial R_{2}} & \frac{\partial r_{C D}}{\partial R_{3}} & \frac{\partial r_{C D}}{\partial R_{4}}\end{array}\right]$

The output variance-covariance matrix $\mathbf{c}_{\mathbf{Y}}$ is a two-dimensional quantity:

$\mathbf{c}_{\mathbf{Y}}\left(r_{A B}, r_{C D}\right)=\left[\begin{array}{ll}c_{11} & c_{12} \\ c_{21} & c_{22}\end{array}\right]=\left[\begin{array}{cc}\sigma_{r_{A B}}^{2} & \operatorname{cov}\left(r_{A B}, r_{C D}\right) \\ \operatorname{cov}\left(r_{C D}, r_{A B}\right) & \sigma_{r_{C D}}^{2}\end{array}\right]$

This matrix can be depicted by an ellipse in the plane [9]. It is the horizontal intersection curve of the Gaussian probability surface with a plane at the level of its inflection point.

Having the covariance, the Pearson's correlation coefficient is defined:

$k=\frac{\operatorname{cov}\left(r_{A B}, r_{C D}\right)}{\sigma_{r_{A B}} \sigma_{r_{C D}}}=\frac{c_{12}}{\sqrt{c_{11}} \sqrt{c_{22}}}$.

Its value is within the range from -1 to 1 . Below, the dependence of covariance from the bridge resistances Eqs. (9-10) and their squared uncertainties Eqs. (11-12) are presented:

$$
\begin{gathered}
c_{12}=c_{21}=\frac{\left(R_{2}+R_{4}\right)^{2}}{\sum R_{i}^{4}}\left(\left(R_{3}+R_{4}\right)\left(R_{2}+R_{3}\right) \sigma_{R 1}^{2}+\left(R_{1}+R_{2}\right)\left(R_{1}+R_{4}\right) \sigma_{R 3}^{2}\right) \\
-\frac{\left(R_{1}+R_{3}\right)^{2}}{\sum R_{i}^{4}}\left(\left(R_{3}+R_{4}\right)\left(R_{1}+R_{4}\right) \sigma_{R 2}^{2}+\left(R_{1}+R_{2}\right)\left(R_{2}+R_{3}\right) \sigma_{R 4}^{2}\right) .
\end{gathered}
$$

If there are two RTDs in the bridge-circuit then $R_{i}=R_{0}+\Delta R_{i}$ (for $i=1,2$ ) and $R_{i}=R_{0}$ (for $i=3,4$ ), when $R_{0}$ is nominal resistance (e.g. $100 \Omega$ ). According to IEC 60751 standard the operating limits of a Pt100 sensor $R_{i}$ can be within the range from 81.48 to $390.48 \Omega$. This corresponds to the temperature range from -200 to $+850^{\circ} \mathrm{C}$. In Fig. 2 the transfer resistances Eq. (4) are presented in a function of $\Delta R_{1}=2 \Delta R_{2}$ within the range of from -80 to $+80 \Omega$.

Also, assume that there are two uncertainties: of sensors $\sigma_{R 1}=\sigma_{R 2}=\sigma_{S e n}$ and of resistors $\sigma_{R 3}=\sigma_{R 4}=\sigma_{R e s}$. In this case output covariance and uncertainties are as follows:

$$
\begin{aligned}
c_{12} & =c_{21} \\
& =\frac{\left(2 R_{0}+\Delta R_{2}\right)^{2}}{\sum R_{i}^{4}}\left(2 R_{0}\left(2 R_{0}+\Delta R_{2}\right) \sigma_{\text {Sen }}^{2}+\left(2 R_{0}+\Delta R_{1}+\Delta R_{2}\right)\left(2 R_{0}+\Delta R_{1}\right) \sigma_{\text {Res }}^{2}\right) \\
& -\frac{\left(2 R_{0}+\Delta R_{1}\right)^{2}}{\sum R_{i}^{4}}\left(2 R_{0}\left(2 R_{0}+\Delta R_{1}\right) \sigma_{\text {Sen }}^{2}+\left(2 R_{0}+\Delta R_{1}+\Delta R_{2}\right)\left(2 R_{0}+\Delta R_{2}\right) \sigma_{\text {Res }}^{2}\right),
\end{aligned}
$$




$$
\begin{aligned}
c_{11} & =\frac{\left(2 R_{0}+\Delta R_{2}\right)^{2}+\left(2 R_{0}+\Delta R_{1}\right)^{2}}{\sum_{i}^{4} R_{i}^{4}}\left(4 R_{0}^{2} \sigma_{\text {Sen }}^{2}+\left(2 R_{0}+\Delta R_{1}+\Delta R_{2}\right)^{2} \sigma_{\text {Res }}^{2}\right), \\
c_{22} & =\frac{\left(2 R_{0}+\Delta R_{2}\right)^{2}}{\sum R_{i}^{4}}\left(\left(2 R_{0}+\Delta R_{2}\right)^{2} \sigma_{\text {Sen }}^{2}+\left(2 R_{0}+\Delta R_{1}\right)^{2} \sigma_{\text {Res }}^{2}\right) \\
& +\frac{\left(2 R_{0}+\Delta R_{1}\right)^{2}}{\sum R_{i}^{4}}\left(\left(2 R_{0}+\Delta R_{1}\right)^{2} \sigma_{\text {Sen }}^{2}+\left(2 R_{0}+\Delta R_{2}\right)^{2} \sigma_{\text {Res }}^{2}\right) .
\end{aligned}
$$

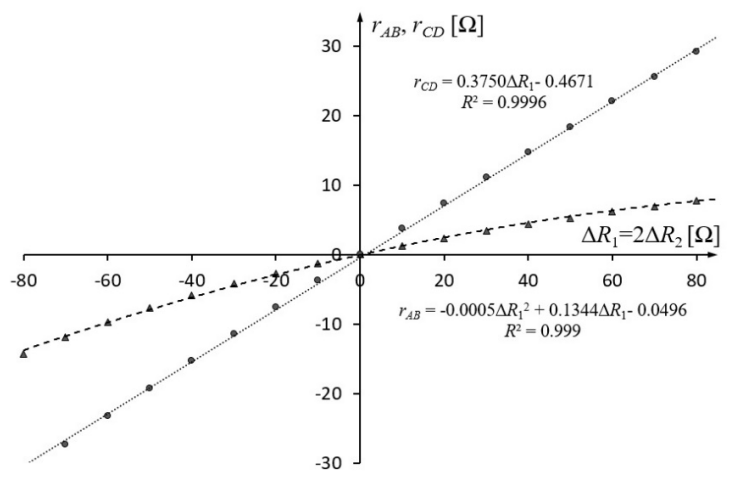

Fig. 2. Dependence of the transfer resistances on the change in resistance when $\Delta R_{1}=2 \Delta R_{2}\left(t_{1}=2 t_{2}\right)$. The temperature difference $t_{D}=t_{1}-t_{2}$ range is limited by nonlinearity of the transfer resistance $r_{A B}$

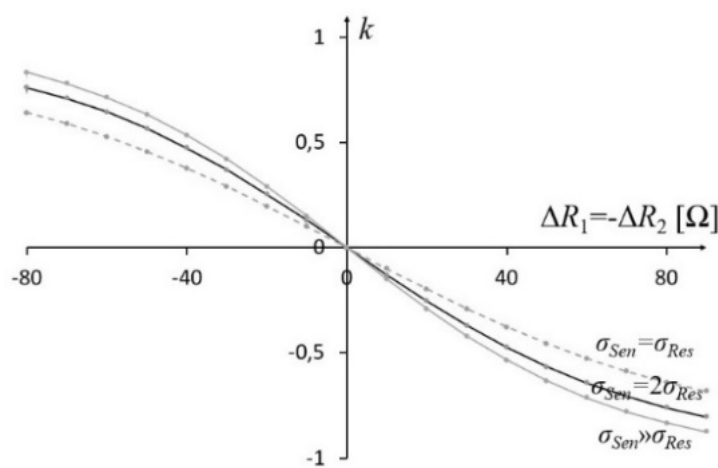

Fig. 3. Dependence of the Pearson's correlation coefficient on the change in resistance when $\Delta R_{1}=-\Delta R_{2}$, different relationships of $\sigma_{S e n}$ and $\sigma_{R e s}$ are assumed. When $\sigma_{\text {Sen }} \gg \sigma_{\text {Res }}$ the correlation (positive and negative) is the highest

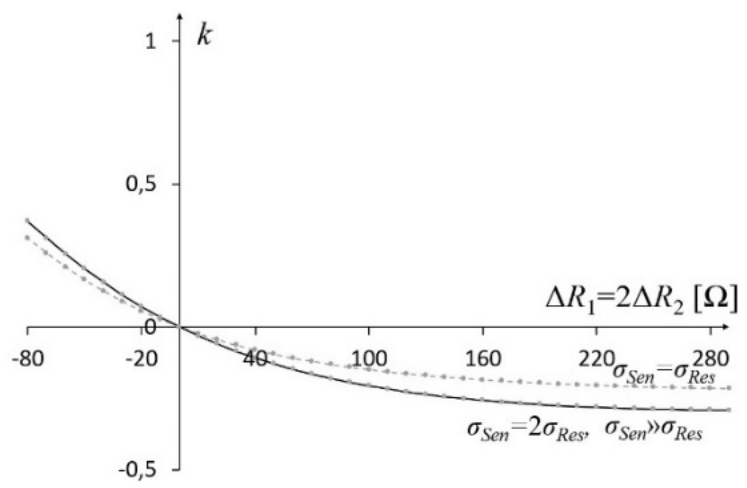

Fig. 4. Dependence of the Pearson's correlation coefficient on the change in resistance when $\Delta R_{1}=2 \Delta R_{2}$. This coefficient does not exceed \pm 0.4 
Fig. 3 presents dependence of correlation coefficient on the change in resistance when $\Delta R_{1}=-\Delta R_{2}$. Fig. 4 depicts it for $\Delta R_{1}=2 \Delta R_{2}$. When $\Delta R_{1}=\Delta R_{2}$ correlation coefficient $k$ is equal to 0 .

\section{Conclusions}

The application shows the usage of variance-covariance matrix for estimating uncertainties of two transfer resistances $\left(r_{A B}, r_{C D}\right)$ of the circuit model. Using Eqs. (4-12) the novel description of the brigde-circuit was created. It develops a wider discussion on the resistance-to-voltage converters and their applications. The maximum nonlinearity error of transfer resistance $r_{A B}$ is $3 \%$ when temperature $t_{1}$ varies from -200 to $200{ }^{\circ} \mathrm{C}$ and temperature $t_{2}$ is half of $t_{1}$ (Fig. 2). Fig. 3 and Fig. 4 depict two variants of relationship between the increments of sensors' resistances when the Pearson's correlation coefficient is different than 0. It shows that both measurement result values should contain standard uncertainties as well as a correlation factor.

\section{References}

[1] D. Ross-Pinnock and P. G. Maropoulos, "Review of industrial temperature measurement technologies and research priorities for the thermal characterisation of the factories of the future," Proceedings of the Institution of Mechanical Engineers, Part B: Journal of Engineering Manufacture, Vol. 230, No. 5, pp. 793-806, May 2016, https://doi.org/10.1177/0954405414567929

[2] Y. Lee, H. Lim, Y. Kim, and Y. Cha, "Thermal feedback system from robot hand for telepresence," IEEE Access, Vol. 9, pp. 827-835, 2021, https://doi.org/10.1109/access.2020.3047036

[3] E. Barajas, X. Aragones, D. Mateo, and J. Altet, "Differential temperature sensors: review of applications in the test and characterization of circuits, usage and design methodology," Sensors, Vol. 19, No. 21, p. 4815, Nov. 2019, https://doi.org/10.3390/s19214815

[4] Sergey Y. Yurish, "A simple and universal resistive-bridge sensors interface," Sensors and Transducers, Vol. 10, No. Special Issue, pp. 46-59, Feb. 2011.

[5] F. Reverter, "The art of directly interfacing sensors to microcontrollers," Journal of Low Power Electronics and Applications, Vol. 2, No. 4, pp. 265-281, Nov. 2012, https://doi.org/10.3390/jlpea2040265

[6] P. J. Sousa, V. C. Pinto, V. H. Magalhães, R. O. Rodrigues, P. C. Sousa, and G. Minas, "Development of highly sensitive temperature microsensors for localized measurements," Applied Sciences, Vol. 11, No. 9, p. 3864, Apr. 2021, https://doi.org/10.3390/app11093864

[7] Z. Warsza, "About accuracy of the simultaneous measurement of temperature difference and average using transducers with Pt sensors," Przemyst Chemiczny, Vol. 1, No. 2, pp. 134-139, Feb. 2017, https://doi.org/10.15199/62.2017.2.20

[8] Z. L. Warsza and A. Idzkowski, "Errors and uncertainties of imbalanced bridge-circuits as primary converters for RTD sensors," Advanced Mechatronics Solutions, Vol. 393, pp. 397-410, 2016, https://doi.org/10.1007/978-3-319-23923-1_60

[9] Jcgm. "JCGM 102: 2011 Evaluation of measurement data - Supplement 2 to the "Guide to the expression of uncertainty in measurement" - Extension to any number of output quantities.". http://www.bipm.org/utils/common/documents/jcgm/jcgm_102_2011_e.pdf (accessed 2021).

[10] P. Fotowicz, "Coverage region for the bidimensional vector measurand," Automation 2017, Vol. 550, pp. 401-407, 2017, https://doi.org/10.1007/978-3-319-54042-9_37

[11] B. Hampel et al., "Approach to determine measurement uncertainty in complex nanosystems with multiparametric dependencies and multivariate output quantities," Measurement Science and Technology, Vol. 29, No. 3, p. 035003, Mar. 2018, https://doi.org/10.1088/1361-6501/aa9d70

[12] Z. L. Warsza and J. Puchalski, "Estimation of uncertainties in indirect multiparameter measurements of correlated quantities," in 2019 12th International Conference on Measurement, pp. 51-57, May 2019, https://doi.org/10.23919/measurement47340.2019.8780042

[13] Z. L. Warsza and A. Idźkowski, "Uncertainty analysis of the two-output RTD circuits on the example of difference and average temperature measurements," in Advances in Intelligent Systems and Computing, pp. 435-446, 2019, https://doi.org/10.1007/978-3-030-15857-6_43 


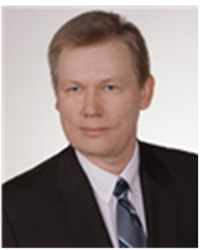

Adam Idzkowski Graduated at Bialystok University of Technology (BTU), Poland. MSc in Electronics and Telecommunications 2001. Ph.D. in electrical engineering 2013. Presently works as associate professor at BTU. Author or co-author above 90 papers in the field of sensors, measurement systems and applications.

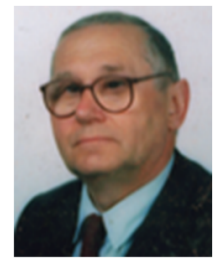

Zygmunt Warsza Graduated at Warsaw Technical University, Poland. MSc. in Electrical Engineering 1959. Ph.D. in El. Measurements 1967. Assoc. Prof. (Docent) from 1970. Presently with Industrial Research Institute of Automation and Measurements (PIAP) in Warsaw, Poland. President of Polish Society of Metrology. Author or co-author above 340 papers, 6 monographs, many reports and 11 patents on new instruments. 